\title{
Synthetic Spectra for Type Ia Supernovae at Early Epochs
}

\author{
D.N. Sauer ${ }^{1}$, A.W.A. Pauldrach ${ }^{2}$, T. Hoffmann ${ }^{2}$, and W. Hillebrandt ${ }^{1}$ \\ 1 Max-Planck-Institut für Astrophysik, Garching, Germany; \\ dsauer@mpa-garching.mpg.de, wfh@mpa-garching.mpg.de \\ 2 Institut für Astronomie und Astrophysik der Universität München, Germany; \\ uh10107@usm. uni-muenchen.de, hoffmann@usm.uni-muenchen.de
}

Summary. We present the current status of our construction of synthetic spectra for type Ia supernovae. These properly take into account the effects of NLTE and an adequate representation of line blocking and blanketing. The models are based on a sophisticated atomic database. We show that the synthetic spectrum reproduces the observed spectrum of 'normal' SN-Ia near maximum light from the UV to the nearIR. However, further improvements are necessary before truly quantitative analyses of observed SN-Ia spectra can be performed. In particular, the inner boundary condition has to be fundamentally modified. This is due to the dominance of electron scattering over true absorption processes coupled with the flat density structure in these objects.

\section{Introduction}

A detailed understanding of the physics and the explosion mechanism of type Ia supernovae (SNe Ia) is essential to minimizing systematic errors of cosmological parameters derived from the interpretation of $\mathrm{SNe}$ Ia observations. A key role in this context play realistic models of the expanding atmospheres of SNe Ia which are based on the full non-equilibrium physics involved, which link the results of current hydrodynamic explosion models (see, e.g., $[1,9]$ ) to the comprehensive observational data.

The spectra of SNe Ia contain useful information about the energetics of the explosion - luminosity and velocity - and the nucleosynthesis, especially in shells above the pseudo-photosphere, which are primarily observable in early epochs. To gain access to this information several groups have started to compute synthetic optical spectra for SNe Ia in the photospheric epoch $[2,3,5,6]$. So far, however, all use simplifying assumptions with uncertain influence on the resulting spectrum. Realistic models for quantitative analysis are still missing.

The main reason we still do not have realistic models is that the physical conditions in expanding SN Ia-atmospheres - high radiation energy density in a low matter density environment - make this modeling especially difficult. Simplifying assumptions such as local thermodynamic equilibrium (LTE) are invalid because radiative processes dominate over local collisional processes. Thus, a realistic model requires at minimum a solution of the full non-LTE 
problem. Other difficulties arise because the ejecta do not contain significant amounts of hydrogen or helium, elements that form the main contribution to continuous opacity in other stellar objects. This causes a strong dominance of line opacity over continuous opacity, which is further complicated by the high expansion velocities of the ejecta (up to $\sim 30000 \mathrm{~km} \mathrm{~s}^{-1}$ ). Thus, thousands of Doppler-shifted and -broadened spectral lines of low-ionized metal atoms overlap and form a non-thermal "pseudo-continuum" with strong lineblocking and line-blanketing effects.

Furthermore, the entire light emission is powered by $\gamma$-photons (and in later times also positrons) originating from the decay of ${ }^{56} \mathrm{Ni} \rightarrow{ }^{56} \mathrm{Co} \rightarrow{ }^{56} \mathrm{Fe}$. These $\gamma$-photons are redistributed to lower energies by scattering and ionization events. They are unlikely to be thermalized - in best case only partially - even within the pseudo-photosphere and the outer-most parts of the atmosphere because the density increases only moderately toward the center. Recent explosion models predict that ${ }^{56} \mathrm{Ni}$ can be present even at high velocities [9]. This indicates the need for a consistent treatment of the energy input within the atmosphere, which may significantly influence the emergent spectrum. Also, the observed changes in the spectra with time as the photosphere recedes into the ejecta show that the abundance of the elements varies strongly with radius.

All these factors have to be taken into consideration to develop a realistic radiative transfer model that allows a reliable quantitative analysis of SNe Ia.

In this paper, we describe the computation of synthetic spectra for SNe Ia based on a consistent treatment of the full NLTE radiative transfer (see [10]). This method will be used to test hydrodynamic explosion models against the observations and will eventually provide a powerful tool for spectral diagnostics of SNe Ia.

\section{The Method}

The basic approach for modeling supernova spectra is analogous to the treatment of O-stars with expanding atmospheres because the physics involved is similar in many respects. Our code development is based on a program package which has proven to be very successful for the quantitative spectral analysis of hot stars with radiation-driven winds $[7,8]$.

The models treat the expanding atmosphere as stationary and spherically symmetric. The underlying density structure, velocity field, and composition of the ejecta (averaged over angles) are provided by the explosion model.

The code provides a consistent solution of the radiative transfer and the NLTE rate equations for the occupation numbers of the atomic levels. A proper treatment of line blocking and blanketing effects is included. All significant contributions to opacity and emissivity from bound-bound, boundfree, and free-free transitions, as well as Thomson-scattering, are taken into account - for this purpose we have compiled and computed a comprehensive 
atomic database involving accurate radiative and collisional ionization and excitation cross-sections and atomic level energies of all important ions.

After each iteration cycle, the temperature for each radius point is determined by balancing all energy gains and losses to the gas - implementation of the $\gamma$-photon deposition is currently under development; thus far, it is only considered with the assumption that all deposition takes place below the photosphere. Together with the use of the diffusion approximation at the lower boundary this implies the assumption of a complete thermalization of the $\gamma$-photons. The total luminosity (given by the amount of synthesized ${ }^{56} \mathrm{Ni}$ and the $\gamma$-deposition efficiency) is a parameter that is used to fit the shape of an observed spectrum.

Finally, the emergent spectrum is given by the computed radiation field at the outer boundary of the converged model.

\section{First Results}

Figure 1 (see [10]) compares the synthetic spectrum of a NLTE-model based on the W7 explosion model [4] to the observed spectrum of SN 1992A. At first glance the spectra seem to agree quite well. However, a closer look reveals a few discrepancies: the model reproduces the UV and blue part of the observed spectrum fairly well, but it fails to match the relatively steep decline toward infrared bands. Additionally, the absorption features of the model that are deeper than the observed may suggest that the peak fluxes are too large.

Thus, we cannot yet consider the models to be sufficient for quantitative spectroscopy of SNe Ia. Relevant SN Ia physics, in particular the treatment of the $\gamma$-deposition and a better analytical approach to the boundary condition at the photosphere (see next section) still have to be included into the model.

\section{Discussion and Conclusions}

The enhanced fluxes of the model to the red of $\sim 4000 \AA$ shown in Fig. 1 result from the invalid assumption of a photosphere analogous to the atmospheres of hot stars.

Various conditions in the ejecta contribute to the breakdown of the diffusion-approximation at the lower boundary. For stellar atmospheres, the diffusion limit for radiation is an excellent approximation as the conditions deep in the photosphere are very close to LTE. This is because the opacities at all wavelengths are dominated by true processes: $\mathrm{H}$ and He provide a strong bound-free continuum and free-free processes are supported by the exponential density increase at the photosphere (see Fig. 2).

In SNe Ia, however, the opacity is dominated by electron scattering over a large wavelength range, between the optical red and the radio band (see Fig. 2) - the sources of true continuous opacity (free-free and bound-free) are 


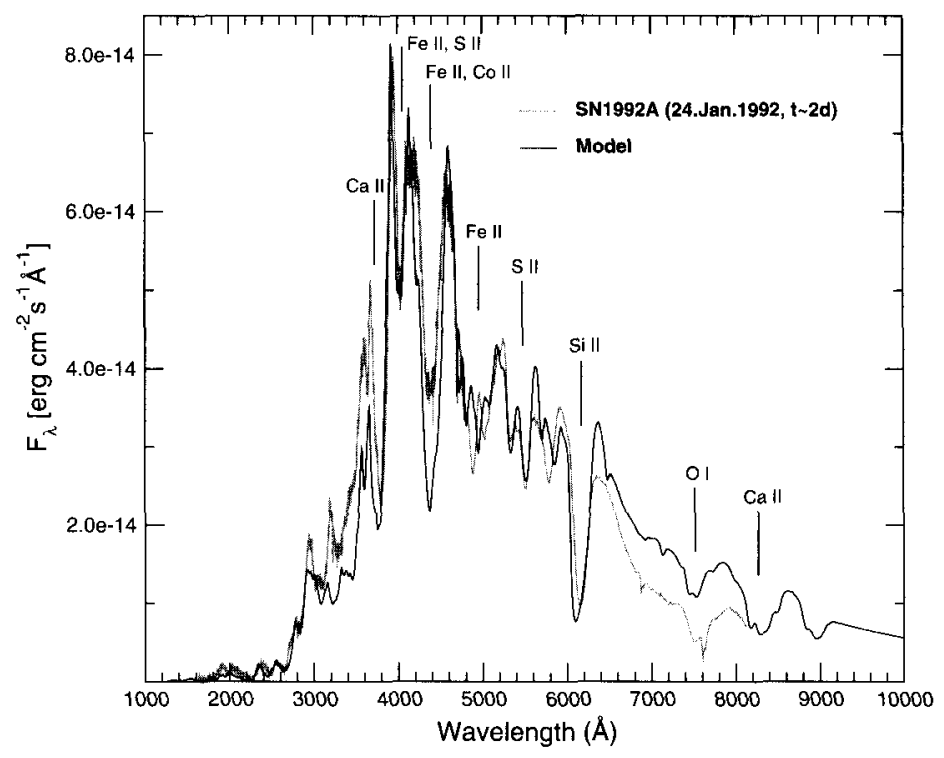

Fig. 1. Synthetic spectrum for a SN Ia around maximum light based on the W7 explosion model with homogenized composition. For comparison, the observed spectrum of SN1992A is also plotted.

orders of magnitude smaller because the composition does not contain hydrogen and helium, and the density distribution is flat. The electron scattering opacity, in contrast to true opacities, does not couple the radiation field to the local temperature. As the flux at the inner boundary from the diffusion approximation is determined by the temperature gradient which is constrained only by the true processes and does not consider scattering dominance. The input flux at these wavelengths is overestimated. We note these conditions do not change at larger optical depths. Moreover, the situation already occurs at early epochs where the assumption of a photosphere is generally considered to be a valid approximation.

These results show that the commonly used lower boundary condition (i.e., analytical expressions based on the diffusion-approximation) is highly problematic for SNe Ia. An inner boundary that correctly accounts for the physical conditions is required in order to correctly solve for the radiation field in SNe Ia.

Overall, the first results obtained with our method are encouraging. The main spectral features of SN Ia spectra are well reproduced, indicating that the underlying physics is correctly described. However, the results also show that even at early epochs the SNe Ia envelopes are comparable neither to the atmospheres of hot stars nor to gaseous nebula. Thus, the assumptions usually made for these cases cannot be applied to the expanding envelopes of 

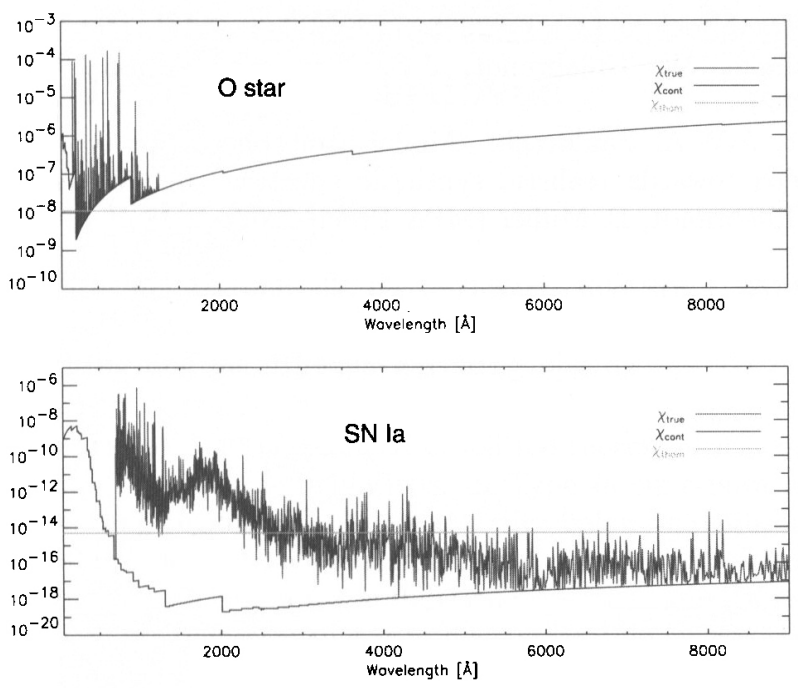

Fig. 2. The contributions to the opacity in a stellar atmosphere (upper panel) and a supernova (lower panel). Shown are the in both cases the Thomson-opacity $\chi_{\text {Thom }}$ (the grey straight line) and the true continuum opacity $\chi_{\text {cont }}$ (the dark solid line). $\chi_{\text {true }}$ denotes the sum of the continuum opacity and the line opacity.

SNe Ia. In particular, a valid analytic approach to the boundary condition at the inner radius of a scattering-dominated atmosphere has to be formulated.

Acknowledgement. We thank our collaborators in the MPA Hydro-Group and the USM Hot-Star group for helpful discussions. This work was supported by the "Sonderforschungsbereich 375-95 für Astro-Teilchenphysik" der Deutschen Forschungsgemeinschaft. Attendance at the meeting was made possible by an EU CORDIS grant provided by the conference organizers.

\section{References}

1. V.N. Gamezo, A.M. Khokhlov, E.S. Oran, A.Y. Chtchelkanova, R.O. Rosenberg: Science 299, 77 (2003)

2. P. Höllich, C.L. Gerardy, R.A. Fesen, S. Sakai: Astrophys. J. 568, 791 (2002)

3. E.J. Lentz, E. Baron, D. Branch, P. Hauschildt: Astrophys. J. 557, 266 (2001)

4. K. Nomoto, F. Thielemann, K. Yokoi: Astrophys. J. 286, 644 (1984)

5. P. Nugent, E. Baron, D. Branch, A. Fisher, P.H. Hauschildt: Astrophys. J. 485, 812 (1997)

6. A.W.A. Pauldrach, M. Duschinger, P.A. Mazzali, J. Puls, M. Lennon, D. Miller: Astron. Astrophys. 312, 525 (1996)

7. A.W.A. Pauldrach, T.L. Hoffmann, M. Lennon: Astron. Astrophys. 375, $161(2001)$ 
8. A.W.A. Pauldrach: astro-ph 0202226 (2003)

9. M. Reinecke, W. Hillebrandt, J.C. Niemeyer: Astron. Astrophys. 391, $1167(2002)$

10. D. Sauer, A.W.A. Pauldrach: "Model atmospheres for type Ia supernovae: basic steps towards realistic synthetic spectra." In: Nuclear Astrophysics, ed. W. Hillebrandt, E. Müller (MPA Proc., 2002) p. 48 